\section{PROBABILITY PERCEPTION}

\section{Why We See Randomness Correctly}

HAHN \& WARREN (2009). Perceptions of randomness: Why three heads are better than four. Psychol Rev, 116, 454.

Kahneman and Tversky (1972, Cogn Psych 3:430) convincingly demonstrated that people incorrectly perceive the randomness of events. Given all possible results of tossing a coin, the likelihood of observing a streak of four heads (HHHH) and a specific nonstreak (e.g., HHHT) are the same. Most people incorrectly judge the streak to be less likely. Hahn and Warren argue, however, that given the experience that people have of random events, this judgment is not only reasonable, but correct! Given one specific result of tossing a coin a finite number of times, the probability of observing a streak is actually less than that of observing a specific nonstreak. The probability of observing $\mathrm{HHHH}$ in a given set of 20 coin flips is only about $50 \%$, whereas the probability of observing HHHT somewhere in that same set is about $75 \%$. So far, that is just math; what is the psychology it leads to? Hahn and Warren suggest that we experience random events with the same kind of temporal sampling that produces this difference in probabilities. The limits of working memory are such that people will likely sample a long sequence of events as experiences of smaller sub-sequences. Basing randomness judgments on these sub-sequences produces an incorrect response for the Kahneman and Tversky task, even if the perception and memory of randomness are correct. - A.E.S.

\section{EXPERIMENTAL CONTROL}

\section{Maintaining Fixation ... With Practice}

Guzman-Martinez et al. (2009). Rapid eye-fixation training without eyetracking. $P B \& R, \mathbf{1 6}, 491$.
Experiments on attention and psychophysics often require observers to maintain fixation to ensure that the results are due to covert attention or to effects at a specific retinal locus. Unfortunately, the typical solutions for minimizing eye movements can be problematic. Eyetrackers are costly (and underutilized if only used to monitor gaze position), and brief timing parameters are not always feasible when studying certain phenomena.

Enter a potential solution: Train observers to maintain fixation. Guzman-Martinez et al. did exactly this by showing observers random dot displays. The display flickered, with all black pixels becoming white, and vice versa. When observers maintained fixation, the display appeared to be uniformly gray because of perceptual averaging; if their eyes moved, they perceived instead a black-andwhite pattern. To train fixation, the authors first had observers perform a spatial-cuing task in which a peripheral attentional cue indicated the probable location of a subsequent target. Not only was eye position monitored, but the experiment used a long delay between the cue and target to allow for eye movements to the cued location. One group of observers was given informed-flicker training: They were asked to maintain fixation and told that seeing a black-and-white pattern indicated that they had made an eye movement. Finally, observers performed the spatial-cuing task again. The informed-flicker training group had reduced the number of eye movements they executed in the task, whereas the control groups showed no such reduction.

These results are important for anyone needing to minimize eye movements in their experiments. Although fixation training does not guarantee that eye movements will not be made, Guzman-Martinez et al.'s training procedure does significantly reduce the likelihood of such movements. Of course, in cases where precise fixation must be maintained, as when making electrophysiological recordings, gaze monitoring will be required. However, using fixation training can minimize the amount of data lost to errant saccades. - S.P.V.

\section{EARLY SPEECH PERCEPTION}

\section{Separating the Wheat From the Chaff}

BEST ET AL. (2009). Development of phonological constancy: Toddlers' perception of native- and Jamaican-accented words. Psychol Sci, 20, 539.

As children begin to acquire a spoken language, such as English, they must learn which differences in pronunciation matter, and which don't, in order to understand spoken words. Differences in dialects and accents, in particular, can significantly change the way spoken words are produced, and children must undercover what properties make a word a word, whether in London or Boise. Best et al. found that this type of perceptual constancy for phonological word forms appears to emerge as a function of development. In a preferential-listening paradigm, 15- and 19-month-old children were presented with familiar and unfamiliar words produced by female speakers from Connecticut and Montego Bay, Jamaica. Younger children were able to recognize familiar words presented in their native Connecticut dialect, but not when the same words were presented in the unfamiliar Jamaican dialect. In contrast, older children were able to recognize familiar words regardless of the dialect, suggesting that by 19 months children have developed phonological constancy. These findings suggest that the ability to cope with variation in pronunciation develops during early childhood, perhaps setting the stage for rapid word learning and vocabulary development. - L.C.N. 


\section{METACOGNITION}

\section{Remember, This Is Not a Competition ...}

Seth (2008). Post-decision wagering measures metacognitive content, not sensory consciousness. Conscious Cogn, 17, 981.

Persaud ET AL. (2008). Commentary to note by Seth: Experiments show what post-decision wagering measures. Conscious Cogn, 17, 984.

You cannot test a poker player's prowess with peanuts; you need cash instead. Persaud et al. (2007, Nat Neurosci 10:257) had cash, and used it to try to deflect criticism of perception without awareness. Instead of merely asking observers whether they were confident about their responses, Persaud et al. invited them to bet on the accuracy of each response. It remains controversial, however, whether such behavior can be used as an index of conscious awareness. In particular, Seth (2008) argued that it is logically impossible to measure awareness in Persaud et al.'s - or any other-single paradigm. Persaud et al. (2008) rebutted this contention, suggesting that their earlier results validate their paradigm. The crux of the controversy is subject G.Y., who failed to place high wagers on better-than-chance responses to subthreshold stimuli placed in his "blindsight" hemifield (Persaud et al., 2007). Seth was willing to consider advantageous wagering as evidence of awareness, or at least of "metacognition," but he dismissed G.Y.'s lack of advantageous wagering as a mere "absence (of) evidence.” Persault et al. (2008) did not address this criticism directly; instead, they reiterated G.Y.'s successful exploitation of suprathreshold stimuli and rhetorically asked why his wagering strategy would change if it did not reflect a difference in awareness? Morgan et al. (1997, Nature 385:401) once suggested that normal observers are reluctant to rate vague-yet-reliable sensations with high confidence. Although G.Y. was reluctant to wager on the basis of his clearly reliable responses, perhaps he was nonetheless conscious of vague sensations, just as Morgan et al.'s normal observers had been. -J.A.S

\section{TIME PERCEPTION}

\section{ERP Analysis of Temporal Assimilation}

Mitsudo ET AL. (2009). Electrophysiological evidence of auditory temporal perception related to the assimilation between neighboring two time intervals. NeuroQuantology, 7, 114.

Various distortions affect the perceived duration of time intervals based on the physical characteristics of the signals marking time. One such distortion is an assimilation effect, also referred to as a time-shrinking illusion. This effect can occur, for instance, when three successive intervals mark two intervals. When the second interval equals $200 \mathrm{msec}$, whether the first interval is $40 \mathrm{msec}$ shorter or longer, the two intervals will be perceived as equal. Mitsudo et al. examined the neural correlates of auditory temporal assimilation by analyzing event-related potentials (ERPs). They observed three ERP signatures consistent with a classical behavioral view of the sources (attention, memory, and decisional processes) of errors in interval discrimination: the classical P300 at Pz, contingent negative variation $(\mathrm{CNV})$ at $\mathrm{Fz}$, and a slow negative component (SNC) at F8. The P300 component is assumed to reflect attention to the first interval, and the CNV component reflects memory processes associated with the retention of the first interval; as the first interval grows longer, the $\mathrm{CNV}$ amplitude grows larger. More important, the SNC at F8 appeared after the stimuli, and Mitsudo et al. argue that this component is related to the participants' temporal judgments - that is, to the decision processes. - S.G.

\section{GAZE TRACKING}

\section{Bringing Saccade Attractors Into Focus}

Kienzle et AL. (2009). Center-surround patterns emerge as optimal predictors for human saccade targets. J Vis, 9, 7. Available at http:// journalofvision.org/9/5/7.
Psychophysical methods are getting more and more subtle and powerful. Springboarding from the method of classification images pioneered by Ahumada and Lovell (1971, J Acoust Soc Am 49:1751) in psychoacoustics and introduced into vision science by Ahumada (1996, Percep 26[Suppl. 1]:18 [Abstract]), researchers are finding new and more penetrating ways to analyze the control of behavior by the multiple features of complex stimuli. A nice example of research on the cutting edge of this effort is Kienzle et al.'s study, in which the authors undertake a nonlinear systems analysis of the process that controls where people spontaneously fixate as they take in a complex scene. They asked observers just to "look around in the scene" shown in each of a series of photos taken at various German zoos while the authors tracked the observers' saccadic fixations. A standard classification-image-type approach to this problem would simply average the patches of image centered at the different fixations. And indeed, if there were a single, fixed image template that your eyes were strongly drawn to on each saccade, such an approach would reveal this saccadeattracting template. However, it seems likely that saccades might be differentially attracted by various patterns and/or repelled by others. The authors use a support vector machine (read the article for details) to derive a function on the space of all $13 \times 13$ pixel image patches that reflects the attractiveness/repulsiveness of different patches to saccades. The final model turns out to be simple: The two primary saccade attractors are a circular, bright-center/dark-surround pattern and its negative, dark-center/ bright-surround pattern. These two saccade-attracting templates, derived directly from the data without any assumptions about the number of templates required or the forms they may take, compose the core of a simple model that accounts well for the data. -C.F.C. 


\section{DEPTH PERCEPTION}

\section{Emotional Arousal Interacts With Depth Perception}

Stefanucci \& Storbeck (2009). Don't look down: Emotional arousal elevates height perception. JEP:G, 138, 131

Emotional arousal is known to influence a wide variety of higherlevel cognitive functions, such as attention, memory, judgment, and decision-making, but relatively little research has investigated the effects of emotional arousal on lower-level processes, such as the perception of distance. This gap may seem surprising to anyone who has ever peered at the ground from a great height, for as many of us know, feelings of fear and anxiety often accompany such experiences. What effects do these emotional states have on one's perception of height, and which aspects of these complex emotional states contribute to perception? Recently, Stefanucci and Storbeck examined this issue by comparing direct (height estimation) and indirect (size estimation) measures of height perception across groups of aroused and nonaroused observers. Of particular interest in this study was the fact that arousal level was manipulated independent of fear by having observers in the high-arousal group view pictures that had previously been judged highly arousing, whereas observers in the low-arousal group viewed pictures that had previously been judged nonarousing. Across four experiments, Stefanucci and Storbeck showed that emotional arousal increased both direct and in- direct estimates of distance, but only when observers looked vertically down at a target from a balcony; similar estimates were unaffected by arousal when the observers looked horizontally "down" a hallway. In addition, Stefanucci and Storbeck showed that emotional arousal modulated the perception of height more than emotional valence (positive vs. negative) did, and that the effects of emotional arousal could be amplified when observers were instructed to personalize the pictures. Overall, these findings indicate that nonperceptual, emotion-based variables can influence our perceptions, although at the present time it is unclear exactly how emotional information interacts with perceptual information within the visual system. More importantly, these findings suggest that the arousal component of the emotional state, not the valence, is what underlies this interaction, and this idea may have implications for the treatment of clinical conditions such as acrophobia. - B.S.G.

\section{OLFACTION}

\section{"I Have't in My Nose Too"}

RIVIERE ET AL. (in press). Formyl peptide receptor-like proteins are a novel family of vomeronasal chemosensors. Nature.

In Shakespeare's Twelfth Night, the question of one character's sanity arises. Another character suggests that they should "Carry his water to the wise woman." His water is his urine and the wise woman is, we may presume, the best diagnostician in the neighborhood. The Elizabethan urinalysis that she would have per- formed would have been based on at least three of the senses: vision, olfaction, and taste (!). Here, our interest lies with olfaction. It has been understood since antiquity that different diseases have different smells and that olfaction might be a useful tool in diagnosis. Olfaction's potential extends beyond the obvious (e.g., a decayed tooth) to diseases like lung and breast cancer. Indeed, a few years ago, there was a flurry of interest in the ability of trained dogs to detect cancer. We will omit the joke about the "cat scan" and the "lab test." The dogs' olfactory decisions were extremely accurate.

The dogs were trained to respond to the smell of cancer. Now a new article in Nature suggests that evolution figured this out long before human medicine. Riviere et al. found that various rodents expressed genes on olfactory receptors for a set of molecules related to disease and immune function. Without going into the molecular biological details, the interesting question is why an untrained mouse might be interested in detecting disease. These olfactory receptors are in the vomeronasal organ, which is an auxiliary olfactory system responsible for detecting pheromones and other socially important chemical signals. The molecules detected by the newly discovered receptors might give warnings about contaminated food. Alternatively, the authors speculate that medical diagnosis might be one more way for the rodent to assess the fitness of a potential mate. Perhaps mice discriminate against a potential partner with a preexisting condition. - J.M.W. 